\title{
The Global Burden of Skin and Subcutaneous Disease: A Longitudinal Analysis from the Global Burden of Disease Study From 1990-2017
}

Rachel L. Giesey, DO', Sino Mehrmal, DO², Prabhdeep Uppal, DO, MS ${ }^{3,4}$, Gregory R. Delost, $\mathrm{DO}^{5,6}$

\author{
${ }^{1}$ Department of Dermatology, University Hospitals Cleveland Medical Center, Cleveland, $\mathrm{OH}$ \\ ${ }^{2}$ Department of Internal Medicine, Alameda Health System - Highland Hospital, Oakland, CA \\ ${ }^{3}$ Department of Emergency Medicine, Christiana Care Health System, Newark, DE \\ ${ }^{4}$ Department of Family Medicine, Christiana Care Health System, Newark, DE \\ ${ }^{5}$ Apex Dermatology and Skin Surgery Center, Mayfield Heights, $\mathrm{OH}$ \\ ${ }^{6}$ Lake Erie College of Osteopathic Medicine, Erie, PA
}

\section{ABSTRACT}

Background: The global prevalence and disability of skin and subcutaneous diseases have grown annually in recent decades. Large-scale epidemiologic data is useful for better characterization of skin disease to create more impactful and sustainable interventions.

Methods: We assessed multiple global trends in skin and subcutaneous disease from 1990 to 2017 in 195 countries worldwide through the latest Global Burden of Disease Study results from 2017.

Results: Skin and subcutaneous disease grew $46.8 \%$ between 1990 to 2017 and is ranked fourth by incidence of all causes of disease. There is global variation in disease burden when stratified by age, sex, geographic regions, and sociodemographic index. Many global regions experience disproportionately elevated disease burden from certain subcategories of skin and subcutaneous disease. Wealthier countries generally experienced the highest age-standardized disability rates of skin and subcutaneous disease.

Conclusion: The incidence, prevalence, and disability of skin and subcutaneous diseases are increasing disproportionately among countries and sociodemographic groups. This data may improve our understanding of skin and subcutaneous diseases to direct funding and resources to reduce global disparities.

\section{INTRODUCTION}

Skin and subcutaneous diseases are highly prevalent and one of the greatest contributors to disease morbidity in the world. In 2013, they were the fourth largest cause of nonfatal disease burden worldwide and ranked 18th in the top global contributors to disease burden. ${ }^{1}$ That same year in the United States alone, one in four individuals across all ages were seen by a physician for at least a single skin condition. ${ }^{2}$ Half of all skin conditions are associated with mortality, and on average, death from skin disease has been seen to occur five years younger (68.2 years) than the average age of death for all causes. ${ }^{2}$ 
Skin and subcutaneous diseases involve the global interaction of a variety of factors that impact regional disease morbidity. Analyzing these trends on a large scale may improve our understanding of the risk factors and characteristics of skin and subcutaneous diseases to direct funding and resources to reduce global disparities through more impactful and sustainable interventions.

One measurement of disease morbidity is disability-adjusted life years (DALYs), measured as years of life lost due to premature mortality in the population plus the years lost due to disability (YLD) for people living with a health condition or its consequences. ${ }^{3,4}$ Furthermore, the sociodemographic index (SDI) was developed in 2016 to track key measures of socioeconomic development, predict health outcomes, monitor inequalities, and monitor the impact of interventions on health outcomes such as DALYs.5,6 The SDI combines income per capita, years of schooling, and total fertility rate (TFR) to identify where countries sit on a spectrum of development on a scale of zero to one..$^{5}$ The TFR is a summary measure of the average number of children a woman would deliver over her lifetime. ${ }^{5}$ One represents the highest possible income per capita, greatest average number of years of schooling, and lowest TFR. ${ }^{5}$ These metrics are useful in helping dermatologists and public health officials formulate and endorse data driven public health policies and guidelines to reduce the global burden of skin and subcutaneous disease.

In this study, we show epidemiological data and multiple global trends in skin and subcutaneous disease. All results are derived from the Global Burden of Disease (GBD) database. We also provide various indicators of global skin and subcutaneous disease disability and how they relate to age, geographic regions of the world, and SDI. Other studies exist describing the global burden of skin and subcutaneous disease using 2010 and 2013 GBD study data. ${ }^{1,7}$ To our knowledge, there have been no further developments that include the most recent 2017 GBD study results. In addition, current GBD literature in skin and subcutaneous diseases have not yet addressed epidemiologic and disease burden trends as they relate to SDI. Our study makes an important contribution to the growing and rapidly changing body of literature addressing global trends in the epidemiology and burden of skin and subcutaneous disease.

\section{METHODS}

Our data was derived from publicly available GBD datasets from 2017. The GBD datasets provide data to compare the magnitude of diseases, injuries, and risk factors across age groups, sexes, countries, regions, and time from 1990 to the present day for over 350 diseases in 195 countries. ${ }^{5} \mathrm{~A}$ list of multiple disease etiologies measured by the GBD (e.g. skin and subcutaneous disease, cardiovascular diseases, neoplasms) with their corresponding global YLD rank and percent change from 1990 to 2017 is provided (Table 1). A similar table was created listing disease etiologies by global incidence rank along with their corresponding percent change from 1990 to 2017 (Table 2).

We provided a world map of the percent change in age-standardized prevalence rate of skin and subcutaneous disease per 100,000 population by country from 1990 to 2017 (Figure 6). Global prevalence metrics for ages ranging from age 0 to $95+$ and the sex categories male and female were also provided for skin and subcutaneous disease 
Table 1: Years lived with disability (YLD) ranks when considering skin and subcutaneous disease collectively from 1990-2017.

\begin{tabular}{|c|c|c|c|}
\hline Cause & 2017 Global YLDs & $\begin{array}{l}\text { Percent Change } \\
(1990-2017)\end{array}$ & 2017 YLD rank \\
\hline Musculoskeletal disorders & $135,881,239$ & $66.0 \%$ & 1 \\
\hline Mental disorders & $122,746,275$ & $49.4 \%$ & 2 \\
\hline Neurological disorders & $73,161,793$ & $59.1 \%$ & 3 \\
\hline Sense organ diseases & $66,576,077$ & $76.2 \%$ & 4 \\
\hline $\begin{array}{l}\text { Other non-communicable } \\
\text { diseases }\end{array}$ & $53,645,926$ & $41.9 \%$ & 5 \\
\hline $\begin{array}{l}\text { Diabetes \& Chronic kidney } \\
\text { disease }\end{array}$ & $45,844,350$ & $117.5 \%$ & 6 \\
\hline Chronic respiratory diseases & $44,311,836$ & $49.7 \%$ & 7 \\
\hline Nutritional deficiencies & $42,376,235$ & $-15.5 \%$ & 8 \\
\hline $\begin{array}{l}\text { Skin and subcutaneous } \\
\text { diseases }\end{array}$ & $41,621,861$ & $40.2 \%$ & 9 \\
\hline Unintentional injuries & $36,509,677$ & $51.5 \%$ & 10 \\
\hline Cardiovascular diseases & $35,697,253$ & $87.7 \%$ & 11 \\
\hline Substance use disorders & $31,052,753$ & $56.6 \%$ & 12 \\
\hline Maternal \& neonatal disorders & $29,894,299$ & $83.2 \%$ & 13 \\
\hline Digestive diseases & $19,939,736$ & $58.0 \%$ & 14 \\
\hline $\begin{array}{l}\text { Neglected topical diseases \& } \\
\text { malaria }\end{array}$ & $13,622,881$ & $-8.1 \%$ & 15 \\
\hline Transport injuries & $13,394,368$ & $61.6 \%$ & 16 \\
\hline $\begin{array}{l}\text { Respiratory infections \& } \\
\text { tuberculosis }\end{array}$ & $11,670,255$ & $27.6 \%$ & 17 \\
\hline Enteric injections & $10,583,692$ & $43.8 \%$ & 18 \\
\hline Neoplasms & $7,775,158$ & $124.0 \%$ & 19 \\
\hline Self-harm \& violence & $7,270,424$ & $43.8 \%$ & 20 \\
\hline $\begin{array}{l}\text { HIV/AIDS \& sexually } \\
\text { transmitted infections }\end{array}$ & $5,369,731$ & $185.7 \%$ & 21 \\
\hline Other infectious diseases & $4,056,632$ & $4.4 \%$ & 22 \\
\hline
\end{tabular}

in 2017 (Figure 1). When looking at the global morbidity of dermatoses as measured in DALYs per 100,000 population in 2017 , comparisons were made to age ranges (Figure 2), and geographic regions of the world (Figure 3). A comparison of global skin and subcutaneous disease change in DALYs per 100,000 population between 1990 and 2017 is also given for the seven GBD super-regions along with the global average (Figure 4). Further comparisons were made between age-standardized DALYs rates from dermatoses in all 195 countries surveyed by the GBD in 2017 with the respective SDI for each country (Figure 5).

\section{RESULTS}

Across all disease categories measured by the GBD, skin and subcutaneous disease ranked ninth in 2017 when measured by YLD, with an annual percent increase from 1990 to 2017 of $40.2 \%$ (Table 1). When ranked by incidence, skin and subcutaneous disease ranked fourth with an annual percent increase of $46.8 \%$ between 1990 to 2017 (Table 2). 
Table 2: Incidence ranks when considering skin and subcutaneous disease collectively from 1990-2017.

\begin{tabular}{|c|c|c|c|}
\hline Cause & $\begin{array}{l}\text { Number of new } \\
\text { cases (1990-2017) }\end{array}$ & $\begin{array}{l}\text { Percent Change } \\
(1990-2017)\end{array}$ & $\begin{array}{l}2017 \text { Incidence } \\
\text { rank }\end{array}$ \\
\hline $\begin{array}{l}\text { Respiratory infections \& } \\
\text { tuberculosis }\end{array}$ & $17,942,622,200$ & $32.9 \%$ & 1 \\
\hline Enteric infections & $6,307,792,414$ & $51.6 \%$ & 2 \\
\hline $\begin{array}{l}\text { Other non-communicable } \\
\text { diseases }\end{array}$ & $4,209,629,187$ & $33.2 \%$ & 3 \\
\hline $\begin{array}{l}\text { Skin and subcutaneous } \\
\text { diseases }\end{array}$ & 4,185,971,292 & $46.8 \%$ & 4 \\
\hline Nutritional deficiencies & $1,186,745,815$ & $32.5 \%$ & 5 \\
\hline Neurological disorders & $1,006,294,496$ & $46.2 \%$ & 6 \\
\hline $\begin{array}{l}\text { HIV/AIDS \& sexually } \\
\text { transmitted infections }\end{array}$ & $769,111,205$ & $42.1 \%$ & 7 \\
\hline Other infectious diseases & $478,720,559$ & $5.5 \%$ & 8 \\
\hline Digestive diseases & $465,978,615$ & $66.1 \%$ & 9 \\
\hline Unintentional injuries & $415,410,278$ & $47.9 \%$ & 10 \\
\hline $\begin{array}{l}\text { Neglected topical diseases \& } \\
\text { malaria }\end{array}$ & $357,652,091$ & $29.4 \%$ & 11 \\
\hline Mental disorders & $336,996,264$ & $46.1 \%$ & 12 \\
\hline Musculoskeletal disorders & $334,744,943$ & $58.1 \%$ & 13 \\
\hline $\begin{array}{l}\text { Maternal \& neonatal } \\
\text { disorders }\end{array}$ & $101,960,798$ & $6.7 \%$ & 14 \\
\hline Cardiovascular diseases & $72,721,168$ & $82.6 \%$ & 15 \\
\hline Transport injuries & $63,920,593$ & $57.4 \%$ & 16 \\
\hline Chronic respiratory diseases & $62,161,350$ & $26.0 \%$ & 17 \\
\hline Substance use disorders & $60,099,555$ & $44.2 \%$ & 18 \\
\hline $\begin{array}{l}\text { Diabetes \& Chronic kidney } \\
\text { disease }\end{array}$ & $43,444,563$ & $88.1 \%$ & 19 \\
\hline Self-harm \& violence & $41,379,417$ & $27.2 \%$ & 20 \\
\hline Neoplasms & $24,361,623$ & $100.6 \%$ & 21 \\
\hline
\end{tabular}

Many geographic clusters of the globe had an overall increase in skin and subcutaneous diseases including North America, the lower half of South America, Western Europe, Eastern Asia, and Oceania (Figure 6). Not all countries exhibited increased prevalence, and many countries scattered throughout Africa and Southeast Asia exhibited the greatest overall decrease in rates of dermatoses. Females experience greater age-specific prevalence rates of skin disease on a global scale until about the age of 70 , where the trend was seen to reverse with a slight male predominance (Figure 1).
Skin and subcutaneous disease DALY rates by age appear to have a bimodal distribution with peaks between 0 to 18 years of age and past 65 years of age (Figure 2). Most skin disease disability during infancy was seen between 28 to 364 days of age. A large peak in the combined DALY rates of all dermatoses is seen immediately after infancy between 1 to 4 years of age, with an overall decrease in morbidity until 35 to 39 years of age before disease burden continues to increase up to $95+$ years of age. 


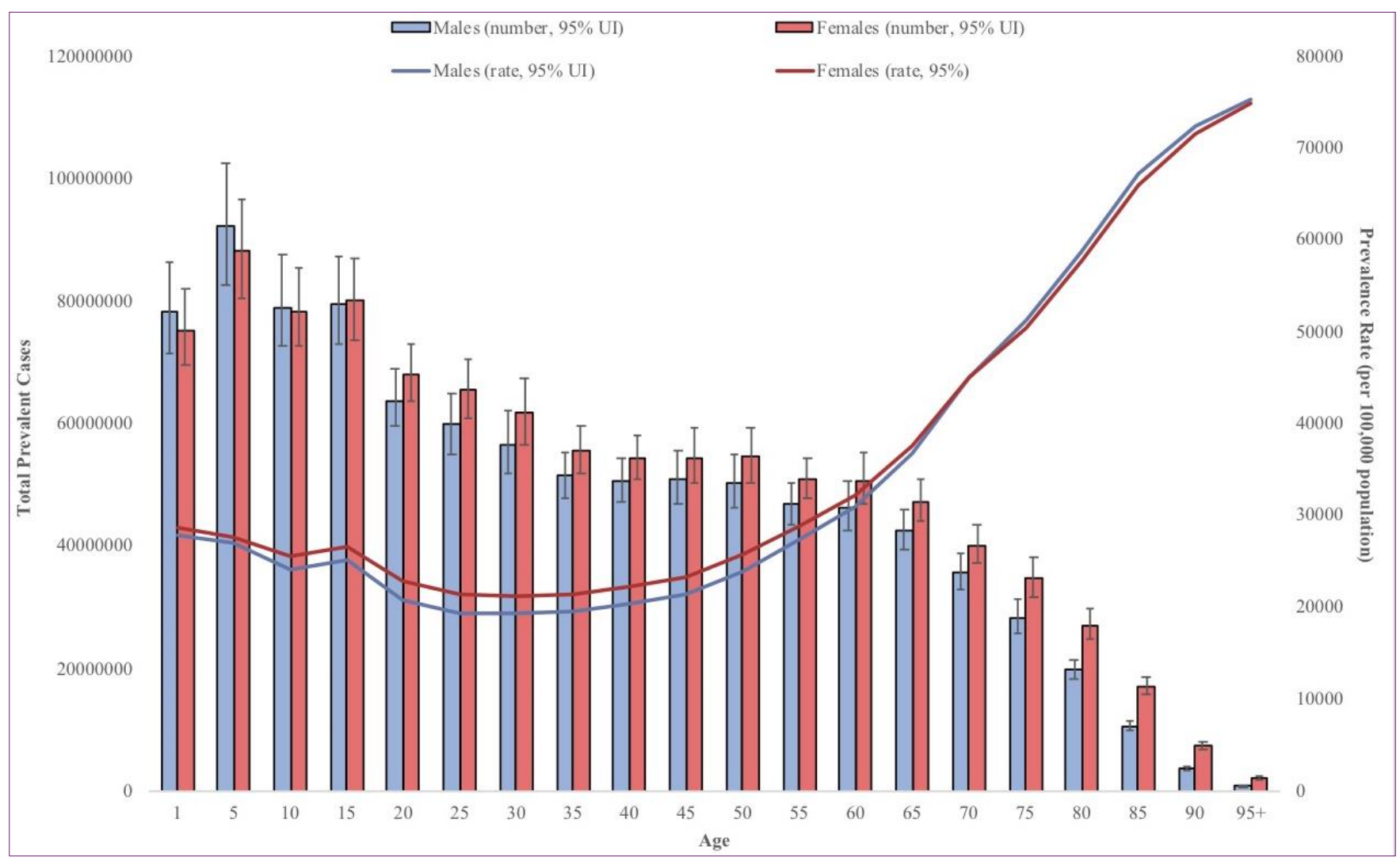

Figure 1. Age patterns by sex in 2017 of the total number of prevalent cases and age-specific prevalence rate of skin and subcutaneous diseases at the global level.

The world regions with the greatest DALY rates in 2017 included high-income North America, Western Europe, and Australasia, while the lowest DALY rates were seen in Central Asia, North Africa and the Middle East, and Central Europe (Figure 3). Three of the seven GBD super-regions ("Highincome," "Sub-Saharan Africa," and "Latin American and Caribbean") exhibited greater DALYs per 100,000 population than the combined global average of all regions (Figure 4). A cluster of high SDI with high age-standardized DALY rates could be seen when comparing age-standardized DALY rates from skin and subcutaneous disease by country and SDI (Figure 5). Generally, a wide variation in age-standardized DALY rates could be seen for other countries along the middle and lower ends of the SDI spectrum.

\section{DISCUSSION}

Both the global prevalence and associated DALYs of skin and subcutaneous disease have grown in recent decades. The global prevalence of all skin disease was $26.79 \%$ in 2017, up from $26.15 \%$ in 1990 , while the percentage of total DALYs due to skin and subcutaneous diseases was $1.76 \%$ in 2017 , up from $1.21 \%$ in $1990 .^{9}$ The top three most prevalent dermatoses were fungal skin diseases (10.09\%); "other skin conditions" encompassing skin diseases such as bullous diseases, connective tissue diseases, and cutaneous drug reactions 


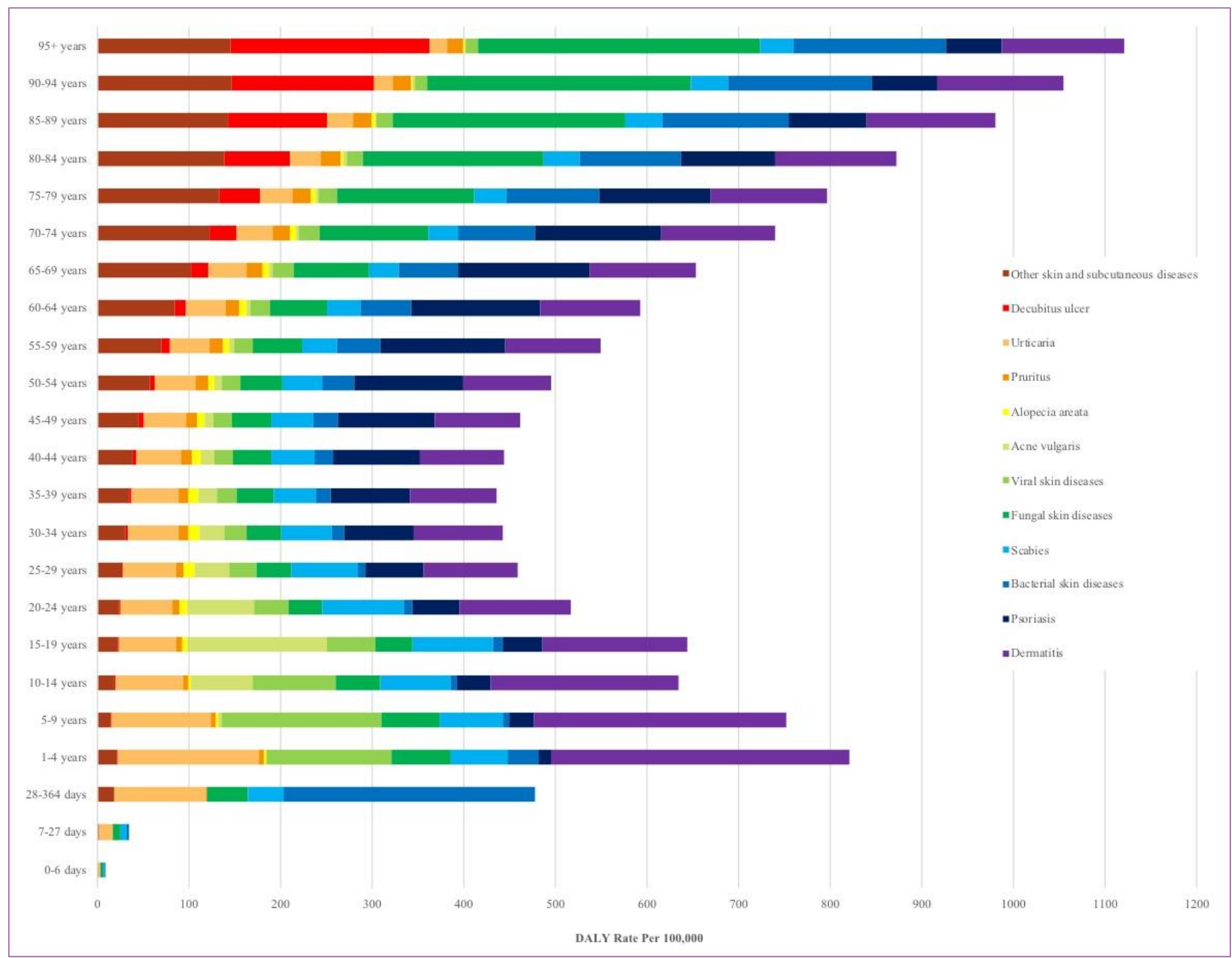

Figure 2. Skin and subcutaneous disease DALY rate per 100,000 at the global level by age, 2017.

$(7.47 \%)$; and atopic dermatitis $(2.79 \%) .9,10$ The top three most disabling dermatoses as measured in DALYs were atopic dermatitis $(0.36 \%)$, psoriasis $(0.22 \%)$, and urticaria $(0.20 \%){ }^{9}$

A few easily treated or preventable dermatoses including fungal skin disease, dermatitis (atopic, contact and seborrheic), and scabies have a disproportionately high loss of productivity and income. ${ }^{10}$ The GBD study diagnoses are arranged according to the International Classification of Diseases (ICD) system, leading to many dermatologic conditions or manifestations grouped in entirely separate disease categories outside the scope of this study. ${ }^{11}$ For example, melanoma and nonmelanoma skin cancer (NMSC) are grouped as "neoplasms", and the entire burden of lupus erythematosus is found in "other musculoskeletal diseases". ${ }^{7}$

Multiple trends regarding the age and sex distribution of skin and subcutaneous diseases have been described. Infants are commonly affected by dermatitis and skin infections; atopic dermatitis is the most common skin disease in children, especially in developed countries, before gradually decreasing with increased age. ${ }^{12}$ Viral warts caused by human papillomavirus ranks 


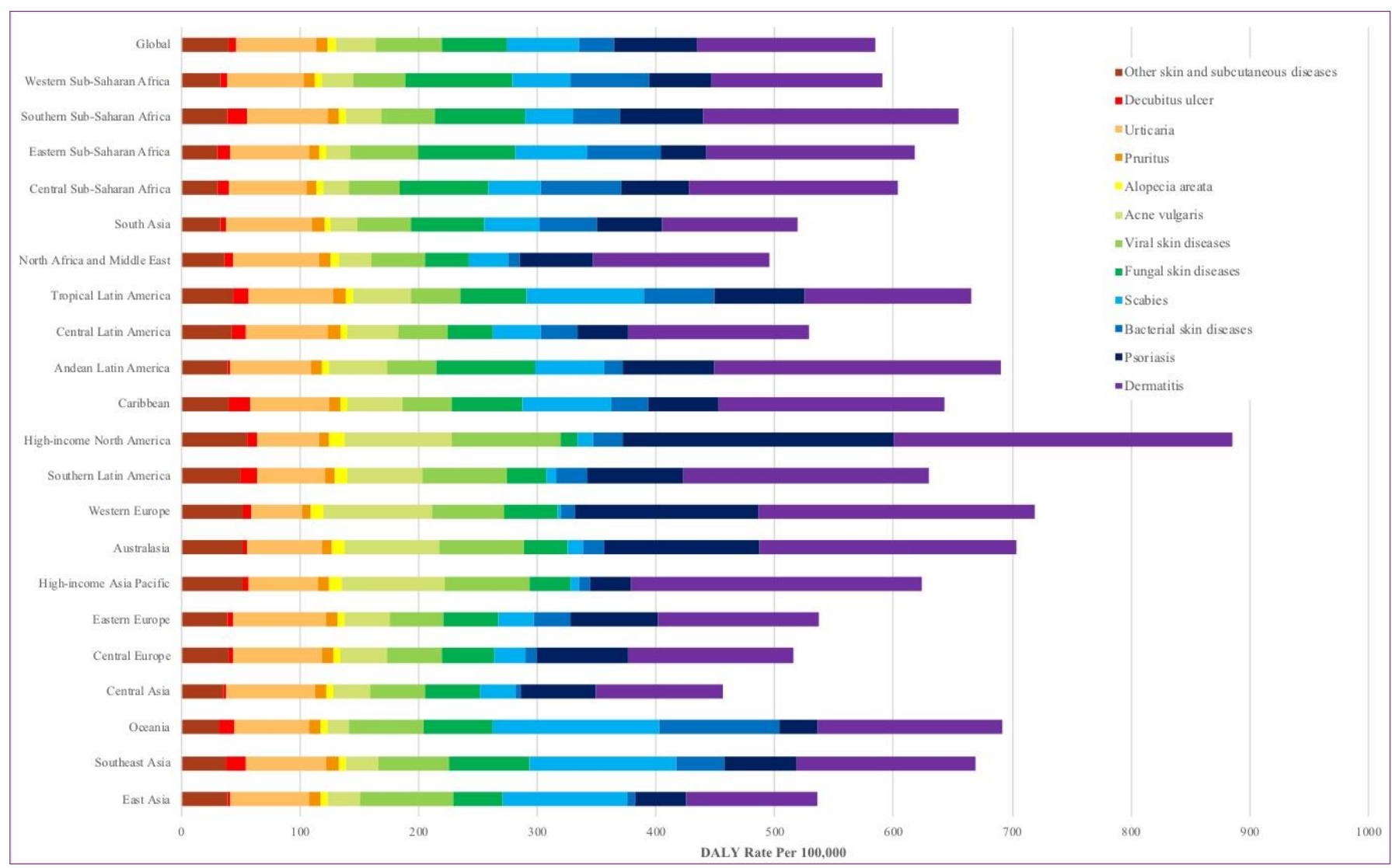

Figure 3. Skin and subcutaneous disease DALY rate per 100,000 by GBD world regions, 2017.

second in children. ${ }^{13}$ Similarly, children in developing countries also generally have high levels of viral warts before prevalence peaks in adolescence and generally resolves by age $16 .{ }^{14}$ Children in developing countries also have greater prevalence of fungal infections such as tinea capitis. ${ }^{15}$

In elderly populations, there is a general predisposition to dermatitis and pressure ulcers due to degenerative skin changes. ${ }^{16}$ Pressure ulcers affect approximately $25 \%$ of the population over the age of 70 and are associated with decreased quality of life, increased rates of disability, and higher treatment costs. ${ }^{17}$ Seborrheic dermatitis prevalence in this population has a male predisposition and may be as high as 31\%, leading to significant distress from pruritus that worsens in the winter. ${ }^{16}$ By $2050,80 \%$ of older populations will be living in low- and middle-income countries. ${ }^{18}$ As the elderly population and corresponding rates of skin and subcutaneous disease continue to grow, an emphasis on primary prevention may serve dual functions of lowering disease burden and serving as a cost-effective intervention for lower income populations.

Wide geographic variation is also characteristic of many skin and subcutaneous diseases and may be attributed to a variety of factors. For example, there appears to be some association in the prevalence of psoriasis and country distance from the equator. ${ }^{19}$ However, the geographic variation of psoriasis remains poorly understood and may be due to a combination of climate, genetics, and environmental factors such as antigen exposure and vitamin D levels. ${ }^{19}$ 


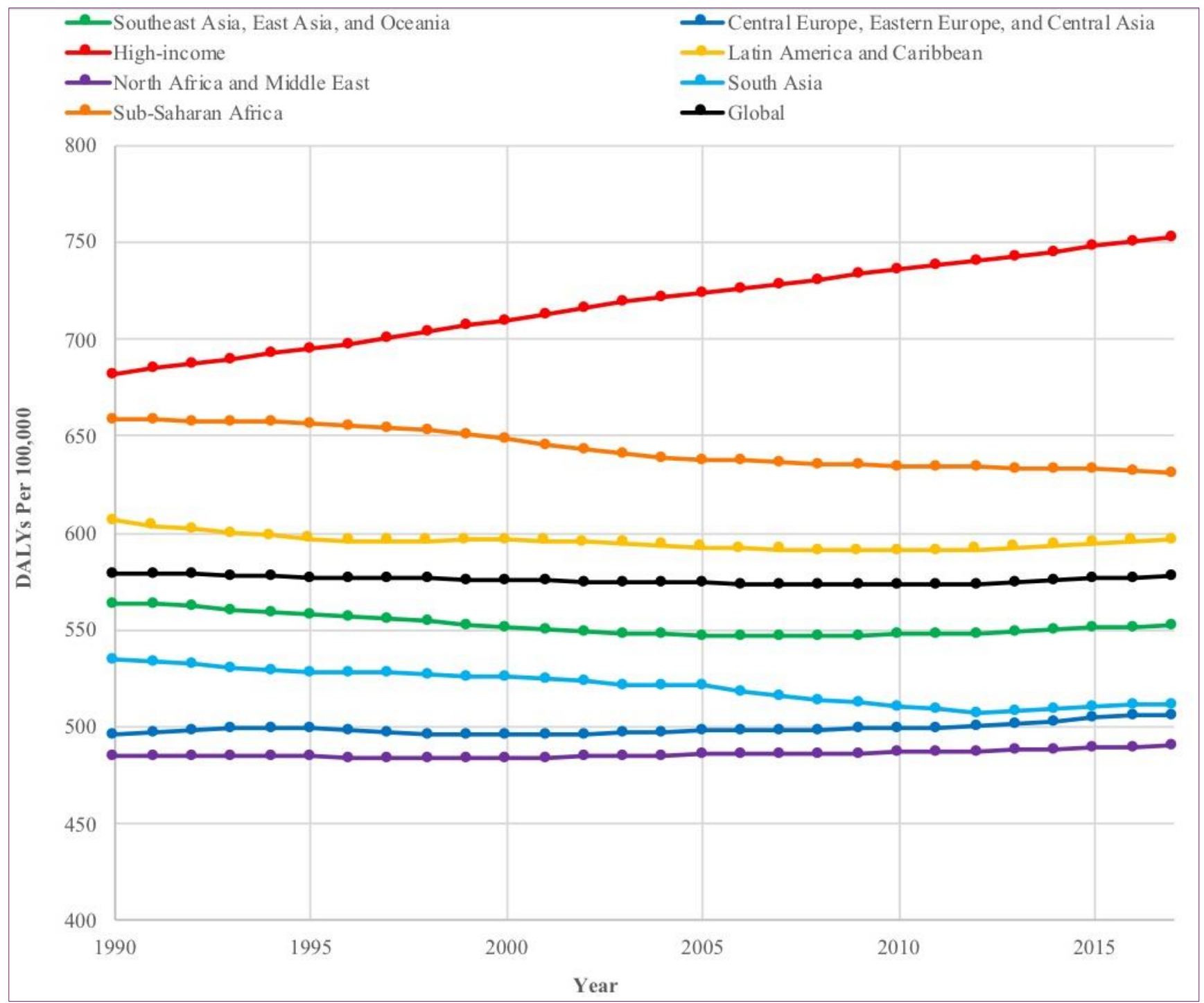

Figure 4. Trends from 1990 to 2017 in DALYs per 100,000 rates of skin and subcutaneous skin diseases in seven GBD super-regions along with the global value.

Geopolitical factors like armed conflict may lead to crowding in refugee camps, thus promoting the spread of transmissible diseases like scabies. ${ }^{20}$ Climate change may also promote variable burden of bacteria, viruses, fungi, and parasites, leading to spread of previously endemic skin diseases beyond their traditional geographic borders. ${ }^{21}$ Socioeconomic factors also affect disease burden as they may dictate the availability and quality of available diagnostics and treatments.
High SDI countries were seen to have high levels of inflammatory dermatoses such as atopic dermatitis and psoriasis. In the case of atopic dermatitis, the hygiene hypothesis has attempted to describe the trend of industrialized countries having higher levels of atopic conditions. ${ }^{22}$ The lack of microbial burden from improved living conditions, antibiotic use, and childhood vaccinations have been seen to promote atypical immune responses. ${ }^{22}$ The risk may be increased further by environmental factors such as increased particle air pollution from motor 


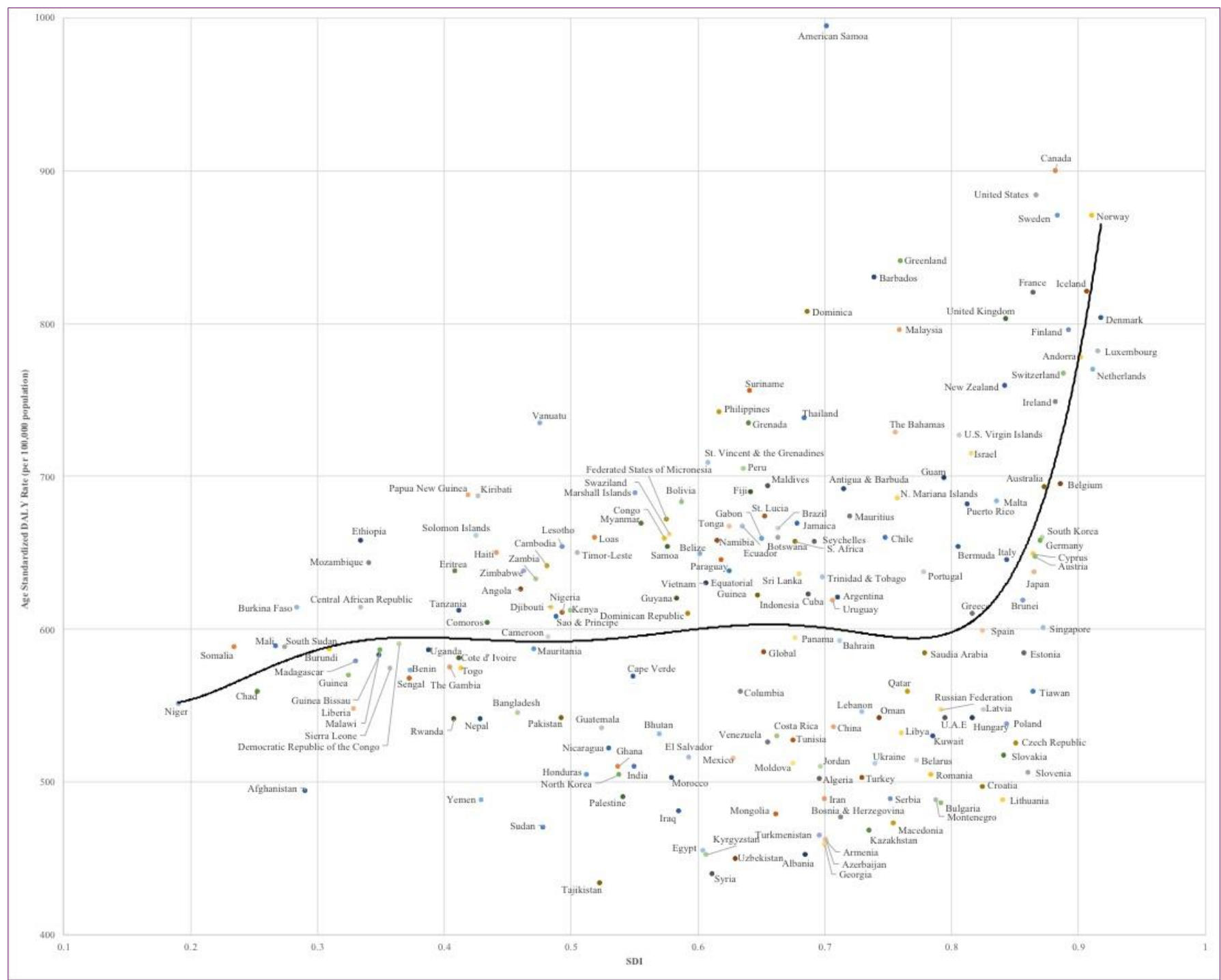

Figure 5. Age-standardized DALYs rates from skin and subcutaneous by socio-demographic index (SDI) for all 195 countries and territories in 2017. Expected values are shown as the black line.

vehicles or second-hand smoke indoors. ${ }^{23}$ There is little data correlating psoriasis with the socioeconomic status of countries, but studies have shown a significant association between poor control of psoriasis and lower educational attainment. ${ }^{24,25}$ Part of the high disease burden in more affluent countries may also be attributed to increased data collection from easier access to physician led specialty care and high sensitivity diagnostics. ${ }^{2,26}$
Though outside the scope of GBD estimates of skin and subcutaneous disease, high skin cancer burden in high SDI populations is a well-studied phenomenon and is a major example of high socioeconomic status driving high disease burden. In the case of skin cancer, high socioeconomic populations are predisposed to increased exposures to occupational chemicals and greater ultraviolet radiation exposure due to easier access to indoor tanning, social pressures of skin appearance, and increased holiday travel. ${ }^{27}$ 


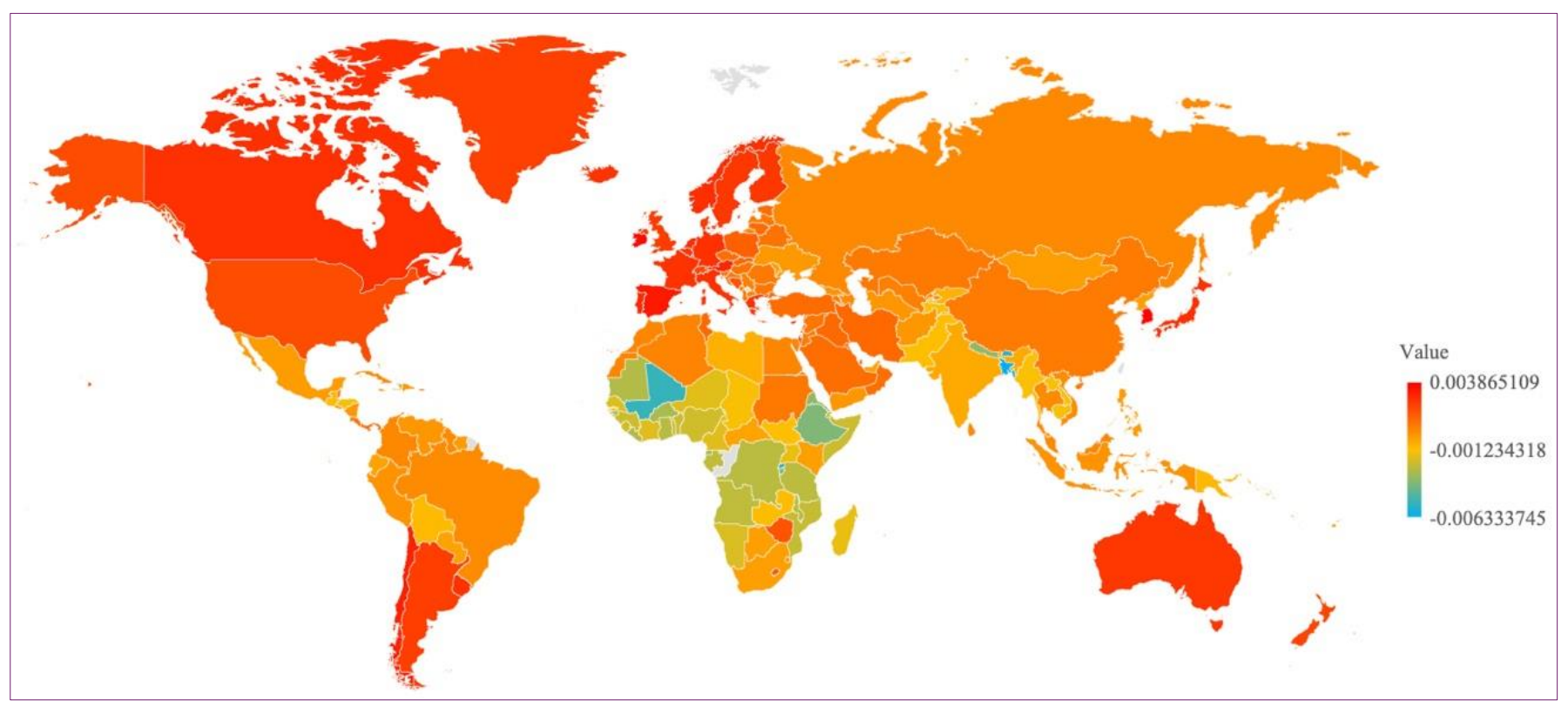

Figure 6. Percent change in age-standardized prevalence rate of skin and subcutaneous disease per 100,000 population from 1990 to 2017. The top ten countries with the largest increase were: Singapore, South Korea, Ireland, Spain, Malta, Cyprus, Chile, Austria, Luxembourg, and Portugal. The top ten countries with largest decrease were: Bangladesh, Rwanda, Bhutan, Mali, Burundi, Nepal, Eritrea, Equatorial Guinea, Comoros, and Burkina Faso.

At the middle and low ends of the SDI spectrum, countries were seen to have higher disease burden from dermatologyrelated infectious diseases including scabies and fungal diseases. High scabies disease burden has been seen in hot, tropical areas as well as overcrowded regions, which may result from armed conflict or natural disaster. ${ }^{20}$ High household density has been shown to be a more important contributor to transmissible skin disease than salary, literacy, shoe use, distance to a water source, and quality of home construction. ${ }^{28}$ Unfortunately, there is little high-quality research on the socioeconomic burden of skin disease in resource-poor settings. Lack of public education and resources may also continue to account for the large number of people continuing to live with disabling skin conditions in these areas, in addition to the lack of physician-led dermatologic care in many low and middle income countries. ${ }^{11,29}$ The lack of research in these regions often leads to extrapolation of findings from high income countries as these countries are often the leaders in shaping global health policies. ${ }^{11}$ Unfortunately, such findings are not always relevant to or feasible in resource poor populations and settings.

Recent estimates of total health care costs of skin disease in the United States were $\$ 75$ billion, with per capita costs of $\$ 240$ annually. ${ }^{2}$ These costs are mitigated by relatively high individual spending power and well developed health systems. Indirect opportunity costs such as missing work are also relatively easier to cope with in high SDI countries. Unfortunately, these are not the features or characteristics of resourcepoor populations and settings. Cost-effective strategies have been described to help bridge such disparities and improve quality of care in resource-poor settings including teledermatology, development of point-ofcare diagnostics, and education of lay persons by experienced providers. ${ }^{11}$ However, increased funding and research is March 2021 Volume 5 Issue 2 
required to account for the different financial, geographic, and cultural variations in resource-poor settings as they relate to skin and subcutaneous disease. ${ }^{11}$

There are limitations when considering the global burden of skin and subcutaneous diseases. As mentioned previously, some dermatologic conditions or manifestations (e.g. melanoma, NMSC, and lupus erythematosus) fall outside the scope of this study as their ICD classifications place them in categories entirely separate from skin and subcutaneous disease, which likely underestimates the true burden of skin disease. ${ }^{7,11}$ Disability estimates may also only reflect symptoms such as itch and appearance changes and may lack the inclusion of complications such as secondary infection or mental health impact. ${ }^{1,11}$

There may also be limitations in geographic diversity where certain populations have a relative excess or scarcity of studies in relation to their total population, which may lead to filling of data gaps through statistical modeling. Additionally, patients may be reluctant to report skin conditions as is commonly seen with the social stigmatization associated with psoriasis, leading to underestimation of disease burden. ${ }^{30}$ Despite the limitations inherent to the GBD and the global reporting of the burden of dermatoses, large scale epidemiological data continues to help dermatologists and key policy and decision makers shape public health policies and better understand skin disease.

\section{CONCLUSION}

Dermatologists will continue to face challenges as they strive to reduce the global burden of skin and subcutaneous diseases. An emphasis on community engagement, outreach, and prevention may lead to more sustainable interventions with greater public health impact. As the growth of our elderly populations outpaces that of other ages, we must be especially cognizant of this vulnerable group with high skin disease burden. We must also keep the accessibility and finances of future interventions in mind to determine their global relevance and feasibility. Increased funding, collaboration with other specialties and health professionals in the care of dermatoses and their comorbidities, and development of new therapies are promising steps to overcome the challenge of reducing the global burden of skin and subcutaneous diseases.
Abbreviations:
DALYs - Disability adjusted life years
GBD - Global Burden of Disease Study
ICD - International Classification of Diseases
NMSC - Non-melanoma skin cancer
SDI - Socio-demographic index
TFR - Total fertility rate

Conflict of Interest Disclosures: The authors have no conflict of interest to declare. This research has been conducted as part of the Global Burden of Diseases, Injuries, and Risk Factors Study (GBD), coordinated by the Institute for Health Metrics and Evaluation. All authors are collaborators with the Global Burden of Disease. This article was not developed with consultation or support with the Global Burden of Disease research team.

Funding: The GBD was partially funded by the Bill \& Melinda Gates Foundation; the funders had no role in the study design, data analysis, data interpretation, or writing of the report.

\section{Corresponding Author:}

Rachel Giesey, DO

Department of Dermatology

University Hospitals Cleveland Medical Center

11100 Euclid Avenue

Lakeside 3500

Cleveland, $\mathrm{OH} 44106$

Phone: 330-592-6091

Fax: 216-844-8993

Email: Rachel.Giesey2@uhhospitals.org 
References:

1. Karimkhani C, Dellavalle RP, Coffeng LE, et al. Global Skin Disease Morbidity and Mortality: An Update From the Global Burden of Disease Study 2013. JAMA Dermatol. 2017;153(5):406-412.

2. Lim HW, Collins SAB, Resneck JS, et al. The burden of skin disease in the United States. J Am Acad Dermatol. 2017;76(5):958-972.e952.

3. Metrics: Disability-Adjusted Life Year (DALY). World Health Organization.

https://www.who.int/healthinfo/global_burden_disease/ metrics_daly/en/. Accessed March 26, 2020.

4. Organization WH. Health statistics and information systems.

https://www.who.int/healthinfo/global_burden_disease/ metrics_daly/en/. Accessed May 26, 2020.

5. Frequently asked questions. IHME. http://www.healthdata.org/gbd/faq. Accessed May 26, 2020.

6. Leach-Kemon K. A new way of measuring development helps assess health system performance. IHME. http://www.healthdata.org/actingdata/new-way-measuring-development-helps-assesshealth-system-performance. Published 2017. Accessed May 26, 2020.

7. Hay RJ, Johns NE, Williams HC, et al. The global burden of skin disease in 2010: an analysis of the prevalence and impact of skin conditions. J Invest Dermatol. 2014;134(6):1527-1534.

8. Protocol for the global burden of diseases, injuries, and risk factors study (GBD). In: Institute for Health Metrics and Evaluation; 2018.

9. Network GBoDC. Global Burden of Disease Study 2017 Results. In: Evaluation IfHMa, ed. Seattle, United States: Global Burden of Disease Collaborative Network. ; 2018.

10. Mehrmal S, Uppal P, Giesey RL, Delost GR. Identifying the prevalence and disability-adjusted life years of the most common dermatoses worldwide. $J$ Am Acad Dermatol. 2020;82(1):258-259.

11. Seth D, Cheldize K, Brown D, Freeman EF. Global Burden of Skin Disease: Inequities and Innovations. Curr Dermatol Rep. 2017;6(3):204-210.

12. Vakirlis E, Theodosiou G, Apalla Z, et al. A retrospective epidemiological study of skin diseases among pediatric population attending a tertiary dermatology referral center in Northern Greece. Clin Cosmet Investig Dermatol. 2017;10:99-104.

13. Nanda A, Al-Hasawi F, Alsaleh QA. A prospective survey of pediatric dermatology clinic patients in Kuwait: an analysis of 10,000 cases. Pediatr Dermatol. 1999;16(1):6-11.

14. Sladden MJ, Johnston GA. Common skin infections in children. BMJ. 2004;329(7457):95-99.

15. Kiprono SK, Muchunu JW, Masenga JE. Skin diseases in pediatric patients attending a tertiary dermatology hospital in Northern Tanzania: a cross-sectional study. BMC Dermatol. 2015;15:16.
16. Farage MA, Miller KW, Berardesca E, Maibach HI. Clinical implications of aging skin: cutaneous disorders in the elderly. Am J Clin Dermatol. 2009;10(2):73-86.

17. Rasero L, Simonetti M, Falciani F, Fabbri C, Collini F, Dal Molin A. Pressure Ulcers in Older Adults: A Prevalence Study. Adv Skin Wound Care. 2015;28(10):461-464.

18. Ageing and health. World Health Organization. https://www.who.int/news-room/factsheets/detail/ageing-and-health. Published 2018. Accessed May 26, 2020.

19. Griffiths CEM, van der Walt JM, Ashcroft DM, et al. The global state of psoriasis disease epidemiology: a workshop report. Br J Dermatol. 2017;177(1):e4-e7.

20. Karimkhani C, Colombara DV, Drucker AM, et al. The global burden of scabies: a cross-sectional analysis from the Global Burden of Disease Study 2015. Lancet Infect Dis. 2017;17(12):1247-1254.

21. Kaffenberger BH, Shetlar D, Norton SA, Rosenbach M. The effect of climate change on skin disease in North America. J Am Acad Dermatol. 2017;76(1):140-147.

22. Okada H, Kuhn C, Feillet H, Bach JF. The 'hygiene hypothesis' for autoimmune and allergic diseases: an update. Clin Exp Immunol. 2010;160(1):1-9.

23. Venn AJ, Yemaneberhan H, Bekele Z, Lewis SA, Parry $\mathrm{E}$, Britton J. Increased risk of allergy associated with the use of kerosene fuel in the home. Am J Respir Crit Care Med. 2001;164(9):1660-1664.

24. Kimball AB, Augustin M, Gordon KB, et al. Correlation of psoriasis activity with socioeconomic status: crosssectional analysis of patients enrolled in the Psoriasis Longitudinal Assessment and Registry (PSOLAR). Br J Dermatol. 2018;179(4):984-986.

25. Mahé E, Beauchet A, Reguiai Z, et al. Socioeconomic Inequalities and Severity of Plaque Psoriasis at a First Consultation in Dermatology Centers. Acta Derm Venereol. 2017;97(5):632-638.

26. Apalla Z, Lallas A, Sotiriou E, Lazaridou E, loannides D. Epidemiological trends in skin cancer. Dermatol Pract Concept. 2017;7(2):1-6.

27. Brouse $\mathrm{CH}$, Hillyer GC, Basch CE, Neugut Al. Geography, facilities, and promotional strategies used to encourage indoor tanning in New York City. J Community Health. 2011;36(4):635-639.

28. Gibbs S. Skin disease and socioeconomic conditions in rural Africa: Tanzania. Int J Dermatol. 1996;35(9):633-639.

29. Tsang MW, Kovarik CL. The role of dermatopathology in conjunction with teledermatology in resource-limited settings: lessons from the African Teledermatology Project. Int J Dermatol. 2011;50(2):150-156.

30. Michalek IM, Loring B, John SM, World Health Organization. Global report on psoriasis. Geneva, Switzerland: World Health Organization; 2016. 